\title{
Visible light effects in plasma plume ignition
}

Cite as: Phys. Plasmas 24, 043502 (2017); https://doi.org/10.1063/1.4979263

Submitted: 13 December 2016 . Accepted: 15 March 2017 . Published Online: 29 March 2017

L. Nie, Y. Xian, (D) X. Lu, and K. Ostrikov
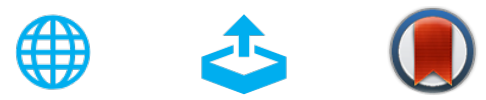

View Online

Export Citation

CrossMark

\section{ARTICLES YOU MAY BE INTERESTED IN}

On the penetration depth of reactive oxygen and nitrogen species generated by a plasma jet through real biological tissue

Physics of Plasmas 24, 073506 (2017); https://doi.org/10.1063/1.4990554

Perspective: The physics, diagnostics, and applications of atmospheric pressure low temperature plasma sources used in plasma medicine

Journal of Applied Physics 122, 020901 (2017); https://doi.org/10.1063/1.4993710

Guided ionization waves: The physics of repeatability

Applied Physics Reviews 5, 031102 (2018); https://doi.org/10.1063/1.5031445 


\title{
Visible light effects in plasma plume ignition
}

\author{
L. Nie, ${ }^{1}$ Y. Xian, ${ }^{1, a)}$ X. Lu, ${ }^{1,2, a)}$ and K. Ostrikov ${ }^{3,4}$ \\ ${ }^{1}$ State Key Laboratory of Advanced Electromagnetic Engineering and Technology, School of Electrical and \\ Electronic Engineering, Huazhong University of Science and Technology, Wuhan, Hubei 430074, \\ People's Republic of China \\ ${ }^{2}$ IFSA Collaborative Innovation Center, Shanghai Jiao Tong University, Shanghai 200240, \\ People's Republic of China \\ ${ }^{3}$ School of Chemistry, Physics and Mechanical Engineering, Queensland University of Technology, \\ Brisbane, QLD 4000, Australia \\ ${ }^{4}$ CSIRO-QUT Joint Sustainable Processes and Devices Laboratory, Commonwealth Scientific and Industrial \\ Research Organization, P.O. Box 218, Lindfield, NSW 2070, Australia
}

(Received 13 December 2016; accepted 15 March 2017; published online 29 March 2017)

\begin{abstract}
The breakdown delay time of a closed plasma plume excited by a high-voltage pulse is investigated. The visible monochromatic light of 404, 532, and $662 \mathrm{~nm}$ wavelength and narrow-waveband light at a central wavelength of 400, 430, 450, 470, 500, 530, 570, 610, and $630 \mathrm{~nm}$ are used to pre-ionize the gas. It is found that the breakdown delay time decreases when the visible light illuminates the discharge tube. The light is most effective when it is applied at the position near the high-voltage electrode. Besides, the tube material and size are important for enhancing the effect. The jet using quartz tube and larger inner diameter make the effect stronger. The effect of visible light is found to inversely relate to the wavelength, manifested by the longer breakdown delay times for longer wavelengths. With increasing the frequency and the pulse width of the voltage, the visible light shortens the delay time more effectively. These observations can be explained by the visible light-enhanced generation of free electrons before the ignition. The proposed mechanisms of free-electron generation are the optically stimulated exoelectron emission from the inner surface of the discharge tube wall and the vibrational excitation of nitrogen molecules. The effects of visible light weaken with the addition of oxygen as a result of electron affinity to oxygen. Published by AIP Publishing.
\end{abstract}

[http://dx.doi.org/10.1063/1.4979263]

\section{INTRODUCTION}

Room-temperature atmospheric-pressure plasma jets (RT-APPJs) have recently attracted significant interest, motivated by their potential applications, such as materials processing, plasma medicine, and chemical decontamination. ${ }^{1-9}$ To understand the physical processes that initiate electrical breakdown of the neutral gas is important for the applications of RT-APPJs. Controlled electrical breakdown is very important in pulsed power applications and represents the basis of the operation of gas-filled switches in fusion reactors, lasers, directed-energy weapons, and electromagnetic pulse generators. Studies found that RT-APPJs have several unique properties including high repeatability. ${ }^{10-13}$ To understand and optimize the ignition stabilization of the discharge, several studies have been reported. ${ }^{10,14}$ Most of these studies focus on the effect of seed electrons. The seed electrons play an important role in the ignition and propagation of the plasma plume and can be produced by cosmic rays, photoionization, and detachment of negative ions. In case of repetitive discharges, residual electrons from the previous discharge pulses can also act as seed electrons.

Photoionization is an effective non-local mechanism for creating free electrons in RT-APPJs. The photons can be generated by photoionization in front of the plasma bullet which

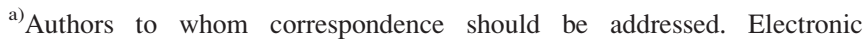
addresses: yubin.xian@hotmail.com and luxinpei@hotmail.com
}

emits UV light. It is commonly believed that only high-energy photons with the energy in the UV or vacuum UV ranges cause the photoionization. Pejović et al. investigated the effect of illumination of nitrogen-filled discharge lamps on nitrogenfilled tubes and found a clear reduction of the breakdown delay time $t_{\mathrm{d}} \cdot{ }^{15}$ One of the possible reasons for the observed decrease of $t_{\mathrm{d}}$ is the enhanced production of the secondary electrons from the cathode induced by the photoelectric effect. Shishpanov et al. studied the breakdown characteristics of a discharge tube illuminated by gas-discharge light sources and found that the tube illumination reduces the breakdown voltage. ${ }^{16}$ They believed that the possible electron source is the optically stimulated exoelectron emission (OSEE) from the inner surface of the discharge tube wall.

Meanwhile, the chemistry of light interactions with the gas may play an important role in the discharge ignition, for example, through vibrational excitation of molecules. ${ }^{17}$ This is due to the fact that the energy is largely transferred from the electrons to the molecular vibration states. Several excited molecules, such as $\mathrm{N}_{2}, \mathrm{CO}, \mathrm{H}_{2}$, and $\mathrm{CO}_{2}$, can maintain their vibrational energy for a relatively long time and without relaxation. This feature is believed to cause selectivity in chemical reactions.

In previous works, the visible light was shown to affect discharge ignition by reducing the breakdown delay time and the breakdown voltage. However, these effects have not been studied systematically for RT-APPJs. Several open questions remain, for example: (1) What is the most effective 
wavelength range of the visible light? (2) Where is the most effective position for the light to be focused on? (3) Does the effect of the visible light depend on the discharge tube size and material? and (4) How does the effect of the visible light depend on the gas composition and pressure.

This work focuses on the effects of visible light on the breakdown delay time of a RT-APPJ. The plasma is generated in a discharge tube. The gas in the tube is replaced after each discharge to eliminate the influence of the residual electrons and reactive species. Visible light of different wave-bands is used to illuminate the discharge tube. The ignition delay time is measured to investigate the effect of the visible light on the discharge. Our results suggest that the breakdown delay time decreases with the visible light illumination and the most effective light wavelength range is $400-530 \mathrm{~nm}$. Besides, this effect is affected by the position of the light source, gas composition, and characteristics of the applied voltage.

The rest of the paper is organized as follows. The experimental setup is described in Section II. The details of the experimental results, including the effects of monochromatic light and the $10 \mathrm{~nm}$ waveband visible light of different wavelengths on the breakdown delay time are presented in Section III. Detailed discussions of the observed phenomena and a brief summary of the results obtained can be found in Section IV.

\section{EXPERIMENTAL SETUP}

The experimental setup is shown in Fig. 1. A customdesigned vacuum-grade gas handling system is used to ensure the purity of the gases and to avoid the diffusion of the surrounding air as shown in Fig. 1(a). The high voltage (HV) electrode and the ground electrode made of a tinfoil are placed on the outside of the tube. The distance between the HV electrode and the ground electrode is $18 \mathrm{~mm}$. It should be pointed out that the system contains no plastic parts, except for the O-ring. The discharge tube is evacuated to $5 \times 10^{-2} \mathrm{~Pa}$ and then back filled with operating gas to a desired pressure. The voltage and current are measured by a HV voltage probe (Tektronix P6015) and a current probe (Tektronix TCP 202), respectively. A photomultiplier tube (PMT, 71D101-CR131) is placed as shown in Fig. 1(a) to evaluate the ignition of the discharge.

Fig. 1(b) shows a schematic of the sequence of the PMT (photomultiplier tube) signals and the voltage pulses. The PMT signals (in Fig. 1(b)) are used to identify the breakdown process. The breakdown delay time $t_{\mathrm{d}}$ is the time between the first voltage pulse and the PMT signals shown in Fig. 1(b). Three lasers with different wavelengths and a xenon lamp are used as the light sources. The three laser sources (with $20 \mathrm{~mW}$ output power) provide monochromatic light of 404, 532, and $662 \mathrm{~nm}$ wavelengths. Besides, the light from the xenon lamp with $30 \mathrm{~W}$ output power together with different narrow-band filters allow light within the selected energy bands to pass through a convex lens to illuminate specific positions along the discharge tube.

\section{EXPERIMENT RESULTS}

It is noticed that visible light illumination can reduce the breakdown delay time. In order to better understand this phenomenon, two kinds of visible light sources, namely the three lasers and narrow-bandwidth visible lights are used to illuminate the gas while the discharge ignition delay time $t_{\mathrm{d}}$ is measured. The optical emission spectra of these light sources (shown in Fig. 2) have been measured by a Princeton Instruments Acton Spectra Hub 2500i spectrometer. All the experiments have been carried out in a dark room to eliminate the interference of any additional light source. Under the same experiment conditions, the measurements have been repeated 20 times. The experiment results from the three lasers are shown in Section III A and the results from the visible light with $10 \mathrm{~nm}$ waveband filters are presented in Section III B.

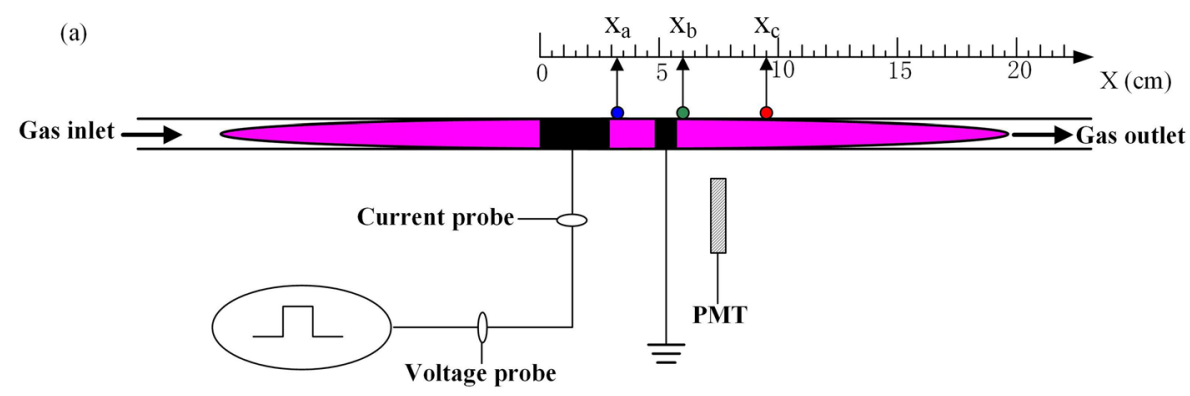

(b)

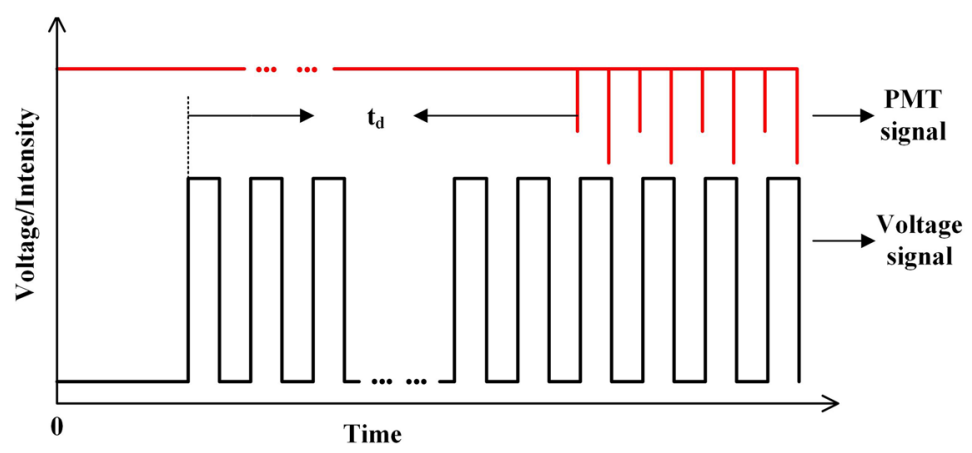

FIG. 1. Schematics of (a) the experimental setup, and (b) the voltage pulses and PMT signals. 

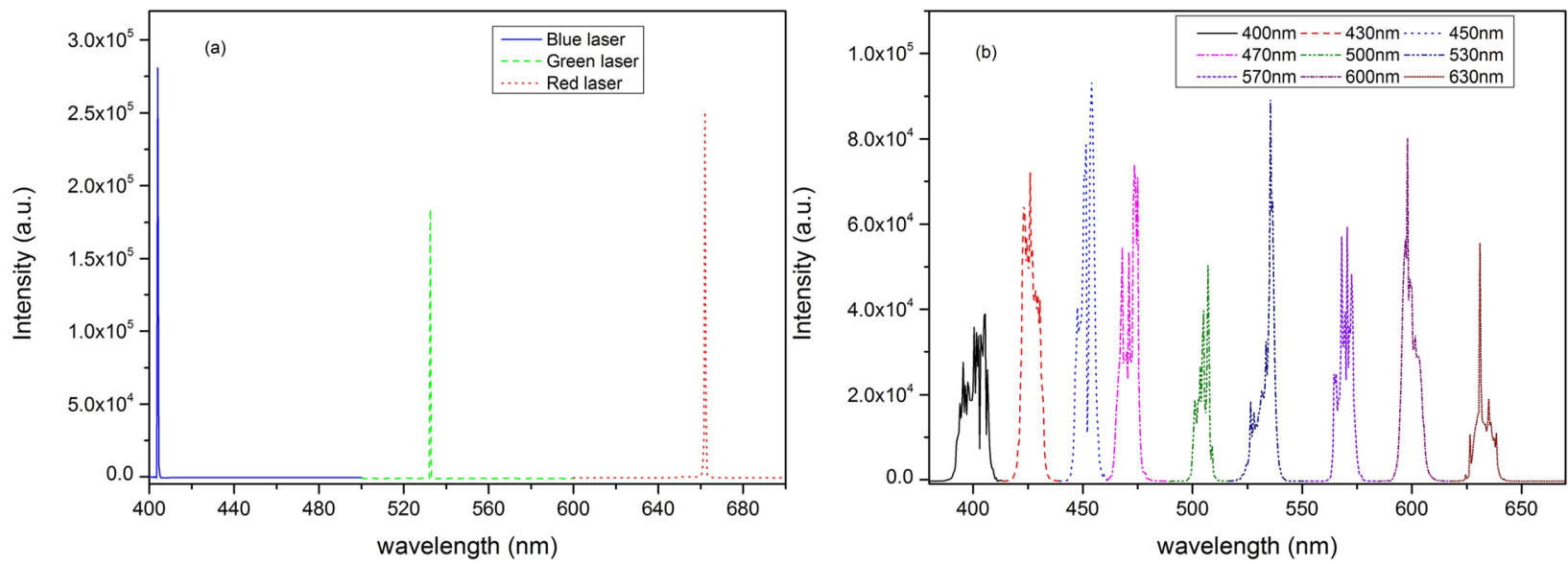

FIG. 2. Emission spectra of (a) the visible light lasers, and (b) the narrow-bandwidth visible light. The grating is $1200 \mathrm{~g} / \mathrm{mm}$, and the entrance and exit slits of the spectroscope are fixed at $50 \mu \mathrm{m}$.

\section{A. The effects of the monochromatic light on the breakdown delay time}

First, the effect of the monochromatic light on the ignition delay time of a helium plasma jet has been studied. In this part, the tube size, material, and gas flow have been changed in order to investigate the effect of the monochromatic light on the breakdown process. And the blue laser with $404 \mathrm{~nm}$ wavelength has been chosen as the illumination source. Helium with purity of $99.999 \%$ is used as a working gas. The voltage, frequency, and pulse width are fixed at $8 \mathrm{kV}, 8 \mathrm{kHz}$, and $2 \mu \mathrm{s}$, respectively. Fig. 3(a) shows the breakdown delay time of plasma jets with different tube size and material when monochromatic light is turned on or off. The parameters of the discharge tube in Fig. 3(a) are listed in Table I. Fig. 3(b) shows the corresponding $t_{\mathrm{d}}$ with different gas flow rates with No. 5 tube.

In Fig. 3(a), it can be seen that the $404 \mathrm{~nm}$ laser decreases the breakdown delay time effectively with 1-5 orders. It should be mentioned that the $t_{\mathrm{d}}$ of No. 2 without additional light is very difficult to be ignited, which means that its $t_{\mathrm{d}}$ is longer than $5 \mathrm{~min}$. The No. 2 jet using Polytetrafluoroethylene tube is difficult to be ignited comparing with the No. 1 jet. By comparing No. 1, No. 3, and No. 4 which have the same wall thickness, the $t_{\mathrm{d}} \mathrm{s}$ are similar without additional light of these tubes, but the $t_{\mathrm{d}}$ of No. 3 with $404 \mathrm{~nm}$ light illuminated on is approximately three orders of magnitude less than that of the No. 1 jet. Therefore, the effect of $404 \mathrm{~nm}$ light on the ignition delay time can be enhanced with larger inner diameter. For No. 4 and No. 5 jets with different wall thickness, the $t_{\mathrm{d}}$ is almost the same with $404 \mathrm{~nm}$ monochromatic light on. From Fig. 3(b), it can be seen that the effect of the monochromatic light on the ignition delay time is independent of the gas flow rate. So, the monochromatic light can reduce the breakdown delay time of RT-APPJs effectively and the effect can be enhanced with large inner diameter with the proper wall thickness and material. And the gas flow rate almost has no influence on this effect.

In the following, the effect of the monochromatic light on the ignition delay time of a helium plasma plume is studied. In this section, the effects of monochromatic light at different laser illumination positions and gas pressures are investigated. And the tube has been connected with the pump in order to avoid the influence of the air and change the parameter of gas. The inner and outer diameters of the discharge tube are 1.5 and $3 \mathrm{~mm}$, respectively.
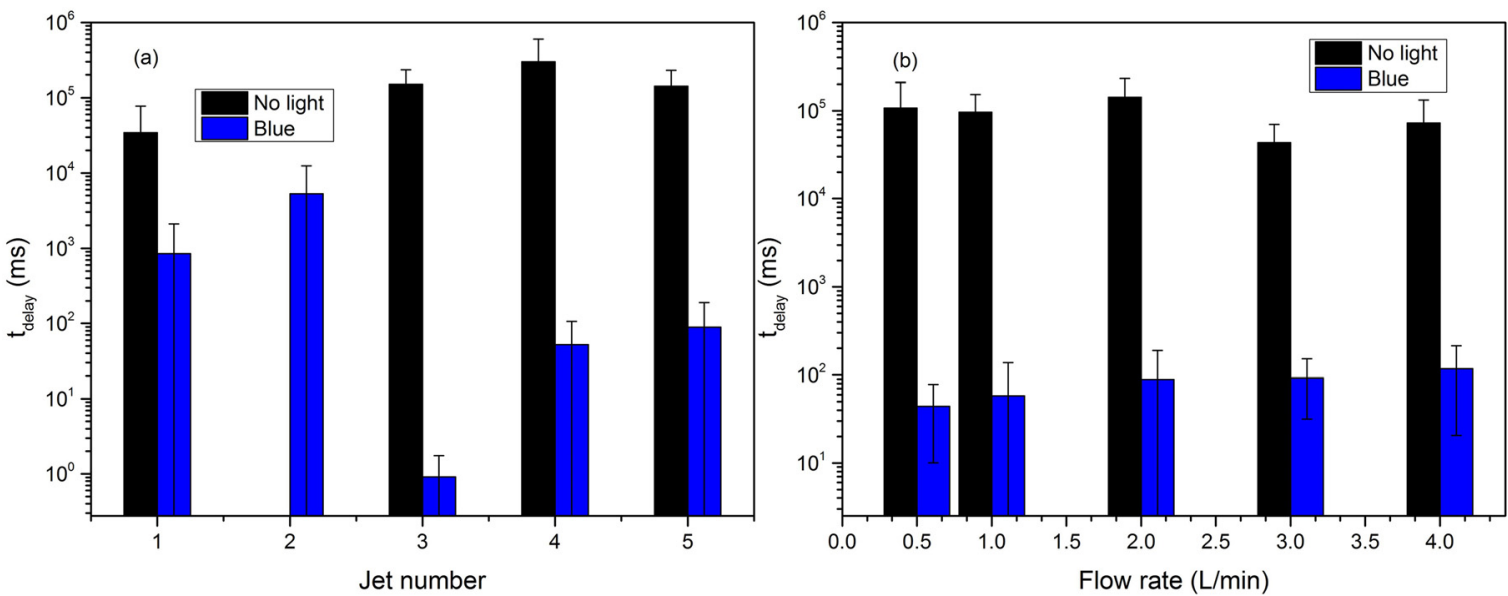

FIG. 3. (a) The delay time of helium with light on or off with different tube size and material according to Table I (flow rate $=21 / \mathrm{min}$ for all cases) and (b) the delay time of helium with light on or off versus gas flow rate. 
TABLE I. The tube size and material of RT-APPJS.

\begin{tabular}{lccc}
\hline \hline No. & Inner diameters $(\mathrm{mm})$ & Outer diameters $(\mathrm{mm})$ & Material \\
\hline 1 & 2 & 4 & Quartz \\
2 & 2 & 4 & Polytetrafluoroethylene \\
3 & 4 & 6 & Quartz \\
4 & 1 & 3 & Quartz \\
5 & 1.5 & 3 & Quartz \\
\hline \hline
\end{tabular}

Helium with purity of $99.999 \%$ is used as a working gas and the gas is still. The voltage, frequency, and pulse width are fixed at $8 \mathrm{kV}, 8 \mathrm{kHz}$, and $2 \mu \mathrm{s}$, respectively. The wavelengths of the monochromatic light in the experiment are 404, 532, and $662 \mathrm{~nm}$. Fig. 4(a)shows the corresponding $t_{\mathrm{d}}$ when the laser illuminates at different positions $\mathrm{x}_{\mathrm{a}}, \mathrm{x}_{\mathrm{b}}$, and $\mathrm{x}_{\mathrm{c}}$ (as shown in Fig. 1) at a gas pressure of $9 \times 10^{4} \mathrm{~Pa}$. Fig. 4(b) shows the breakdown delay time $t_{\mathrm{d}}$ with (or without) light at the pressure of $2 \times 10^{3}, 3 \times 10^{4}$, and $9 \times 10^{4} \mathrm{~Pa}$.

As can be seen from Fig. 4(a), the ignition delay time depends on both the wavelength and the position where the light illuminates. The ignition delay time $t_{\mathrm{d}}$ reduces significantly for all the three lasers when the light illuminates at the position $\mathrm{x}_{\mathrm{a}}$, which is close to the HV electrode. When the blue laser is used, $t_{\mathrm{d}}$ reduces to about $40 \mathrm{~ms}$ which is about four orders less than in the case with no light. When the laser illuminates at the position $\mathrm{x}_{\mathrm{b}}$ which is close to the ground electrode, $t_{\mathrm{d}}$ reduces by about two orders of magnitude for the blue laser, and one order of magnitude for the green laser. For the red laser, the ignition delay time $t_{\mathrm{d}}$ is almost the same as in the case of no light. When the illumination position moves further right to the point $\mathrm{x}_{\mathrm{c}}$ which is about $3.5 \mathrm{~cm}$ away from the ground electrode, illumination by both green and red lasers have no effect on $t_{\mathrm{d}}$. Only when the blue laser is used, $t_{d}$ reduces by about one order of magnitude.

On the other hand, as can been seen from Fig. 4(b), $t_{\mathrm{d}}$ increases when the gas pressure is increased. The blue light laser of $404 \mathrm{~nm}$ significantly reduces the discharge delay time by about four orders of magnitude for all the three gas pressure conditions. The green and the red lasers are less effective.

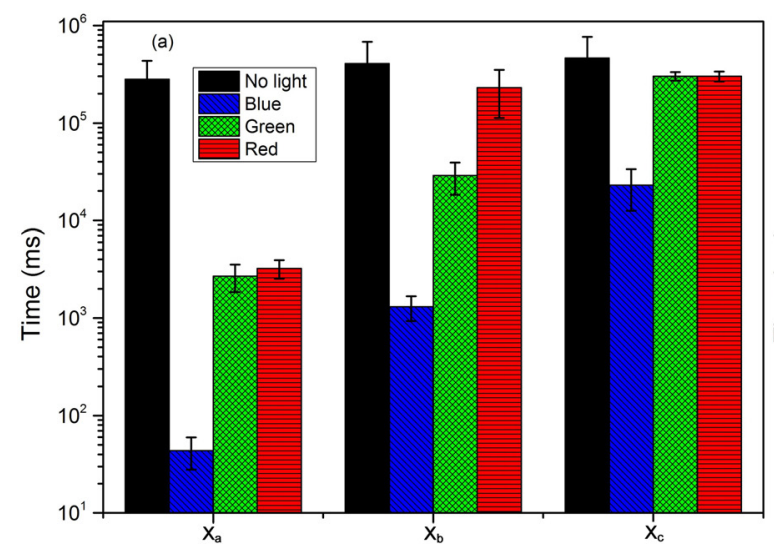

The position of lights illuminate on the tube
As shown above, illumination by all the three visible lasers, especially by the blue laser, reduces the ignition delay time effectively. The photon energy $E$ can be calculated from the equation

$$
E=h c / \lambda
$$

where $h$ is the Planck's constant $\left(4.14 \times 10^{-15} \mathrm{eV}\right), c$ is the speed of light in vacuum, and $\lambda$ is the photon's wavelength. For the lasers with the wavelength of 404,532 , and $662 \mathrm{~nm}$, their photon energy is $3.0688,2.3305$, and $1.8728 \mathrm{eV}$, respectively. These energies are much lower than the first ionization energy threshold of $\mathrm{He}, \mathrm{N}_{2}$, and $\mathrm{O}_{2}$, which are 24.6, 15.58, and $12.2 \mathrm{eV}$, respectively. In other words, the photon energy of the laser light used in the experiment is much lower than the direct ionization energy threshold of the gases. The possible reason for the effect of the lasers on the ignition delay time will be discussed in Section IV.

Meanwhile, as the development of the electron avalanche starts from the HV electrode, the seed electrons around the HV electrode can contribute to the development of the electron avalanche. That might be the reason why the effect of the laser on the delay time depends on the position where the laser illuminates.

\section{B. The effects of visible light with $10 \mathrm{~nm}$ bandwidth on the breakdown delay time}

As shown above, even visible laser light has a significant effect on the breakdown delay time. To further understand the observed effect, light filtered from the xenon lamp has been chosen as the light source as shown in Fig. 2(b). The central wavelengths of the optical filter are 400, 430, $450,470,500,530,570,610$, and $630 \mathrm{~nm}$. In addition, different voltage parameters and gas compositions have been chosen to investigate the effect of the visible light on the breakdown delay time. The inner and outer diameters of the discharge tube are 1.5 and $3 \mathrm{~mm}$, respectively.

Fig. 5 shows the ignition delay times of the discharges illuminated with different center wavelength light measured at the position $\mathrm{x}_{\mathrm{a}}$. Helium of $99.999 \%$ purity is used as a working gas at a pressure of $10^{4} \mathrm{~Pa}$. The amplitude of the

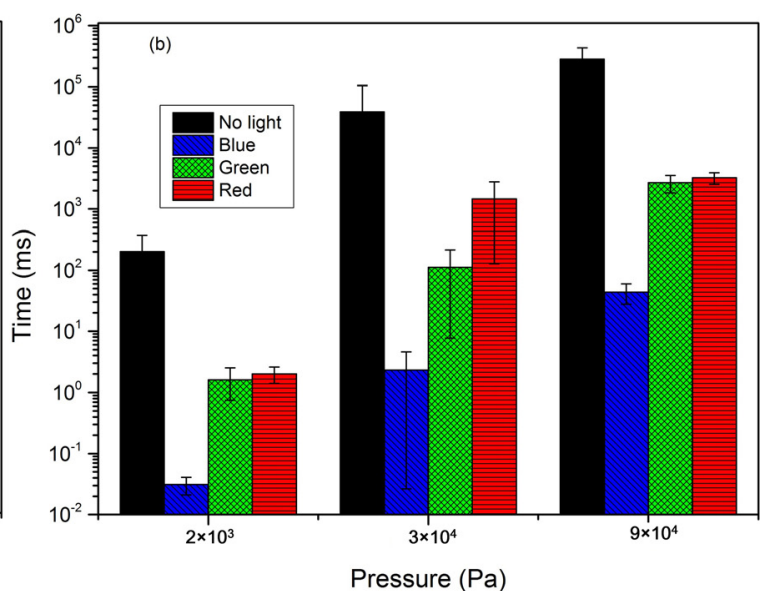

FIG. 4. (a) The values of $t_{\mathrm{d}}$ when laser illuminates at different positions $\mathrm{x}_{\mathrm{a}}, \mathrm{x}_{\mathrm{b}}$, and $\mathrm{x}_{\mathrm{c}}$; gas pressure: $9 \times 10^{4} \mathrm{~Pa}$; (b) the delay time of helium with light on or off at pressures $2 \times 10^{3}, 3 \times 10^{4}$, and $9 \times 10^{4} \mathrm{~Pa}$. 

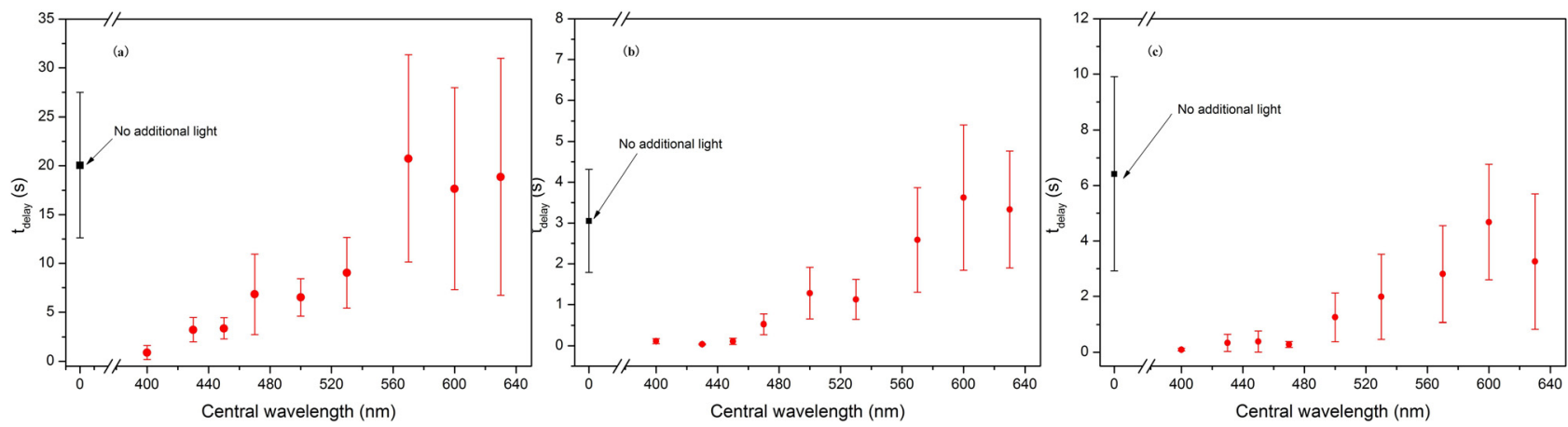

FIG. 5. The ignition delay times of the helium discharge under illumination with narrow-bandwidth light with different center wavelengths:(a) $8 \mathrm{kV}, 2 \mathrm{kHz}$, $2 \mu \mathrm{s}$; (b) $8 \mathrm{kV}, 8 \mathrm{kHz}, 2 \mu \mathrm{s}$; and (c) $8 \mathrm{kV}, 2 \mathrm{kHz}, 10 \mu \mathrm{s}$.

applied voltage is fixed at $8 \mathrm{kV}$. As can be seen from Fig. 5 (a), light with the central wavelength from $400 \mathrm{~nm}$ to $530 \mathrm{~nm}$ can significantly reduce the delay time. However, it was found that the light with the central wavelengths exceeding $530 \mathrm{~nm}$ has no obvious effect. When the frequency is increased to $8 \mathrm{kHz}$, the ignition delay time decreases several folds in all the cases, as shown in Fig. 5(b), even without illumination. However, the observed trend remains the same: the ignition delay time is much shorter when it is illuminated by light with the center wavelengths shorter than $530 \mathrm{~nm}$. A similar trend is observed when the pulse width of the applied voltage is increased to $10 \mu \mathrm{s}$.

We reiterate that pulsed voltage is used in this work. For each voltage pulse, the actual voltage on time $t_{\mathrm{v} \text {-on }}$ is very short compared with the pulse period. For the case of frequency of $2 \mathrm{kHz}$ and pulse width of $2 \mu \mathrm{s}$, the pulse period is $500 \mu \mathrm{s}$. Thus, the actual voltage on time is only $0.4 \%$. With the increase of the pulse frequency or the pulse width, for the same delay time, the actual duration $t_{\mathrm{v}-\mathrm{on}}$ of the high voltage applied to the electrode increases. For example, when the frequency is increased from 2 to $8 \mathrm{kHz}$, the $t_{\mathrm{v} \text {-on }}$ experiences a four time increase if the delay time remains the same. Similarly, when the pulse width is increased from 2 to $10 \mu \mathrm{s}$, the $t_{\mathrm{v}-\mathrm{on}}$ is increased five times at the same delay time.

Therefore, according to Figs. 5(a)-5(c), the ignition delay time is about $20.05,3.05$, and $6.42 \mathrm{~s}$, respectively, when there is no light illumination at all. According to the discussion above, the actual voltage on time $t_{\mathrm{v} \text {-on }}$ is about $80.2,48.8$, and $128.4 \mathrm{~ms}$, respectively, for the three cases when there is no light illumination. In other words, when the frequency is $8 \mathrm{kHz}$, the ignition delay $t_{\mathrm{v} \text {-on }}$ is the shortest. When the $400 \mathrm{~nm}$ light is used, the ignition delay times decrease to about $0.89267,0.11249$, and $0.0947 \mathrm{~s}$ according to Figs. 5(a)-5(c) for the three cases, respectively. The corresponding delay times $t_{\mathrm{v} \text {-on }}$ are $3.57,1.8$, and $1.89 \mathrm{~ms}$, respectively. In other words, high frequencies or long pulses could reduce the observed ignition delays.

Furthermore, the ignition delay time for different gases and gas mixtures has been investigated. The ignition delay time for He mixed with one percent of $\mathrm{N}_{2}$ and $\mathrm{O}_{2}$ is shown in Fig. 6. Similar results for pure $\mathrm{N}_{2}$ and $\mathrm{O}_{2}$ gases are shown in Fig. 7.

One can see from Fig. 6(a) that the delay time for the pure helium without light illumination is about 16 times longer compared with that in Fig. 5(b). Moreover, the light with the central wavelengths ranging from 400 to $630 \mathrm{~nm}$ contributes to the decrease of the delay time. The most effective wavelength range is $400-530 \mathrm{~nm}$ and the effect becomes less obvious when the central wavelength is larger than $530 \mathrm{~nm}$. This phenomenon is almost the same as for the pure helium cases shown in Fig. 5(b).

For the case of $1 \%$ oxygen admixture in Fig. 6(b), the delay time is larger than for the case of $5 \mathrm{~min}$ without light. Moreover, the delay decreases to $10-150 \mathrm{~s}$ when the central wavelength increases from 400 to $470 \mathrm{~nm}$. However, the effect of light with the central wavelength larger than $470 \mathrm{~nm}$ appears to be insignificant.

Therefore, the effective range of narrow-wavelength visible light is much larger for the $1 \%$ nitrogen ad mixture case compared with the $1 \%$ oxygen case. Due to the addition of oxygen, the ignition delay time increases dramatically for all cases. This can be attributed to the electronegativity of
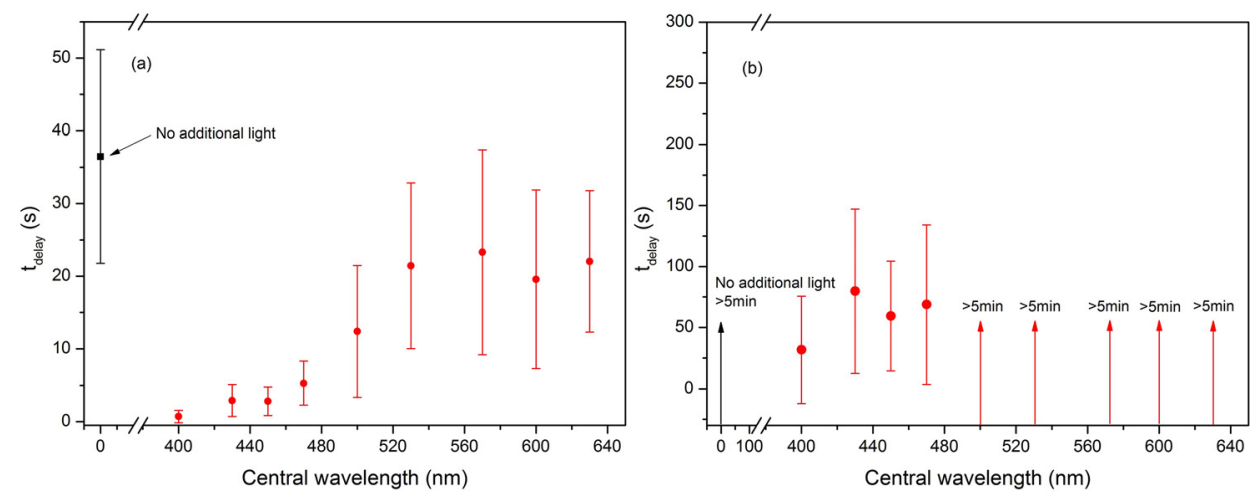

FIG. 6. The ignition delay times for (a) $\mathrm{He}+1 \% \mathrm{~N}_{2}$ and (b) $\mathrm{He}+1 \% \mathrm{O}_{2}$ gas mixtures when light with different central wavelengths is used at a total gas pressure of $10^{4} \mathrm{~Pa}$. The voltage, frequency, and pulse duration are $8 \mathrm{kV}$, $8 \mathrm{kHz}$, and $2 \mu \mathrm{s}$, respectively. 

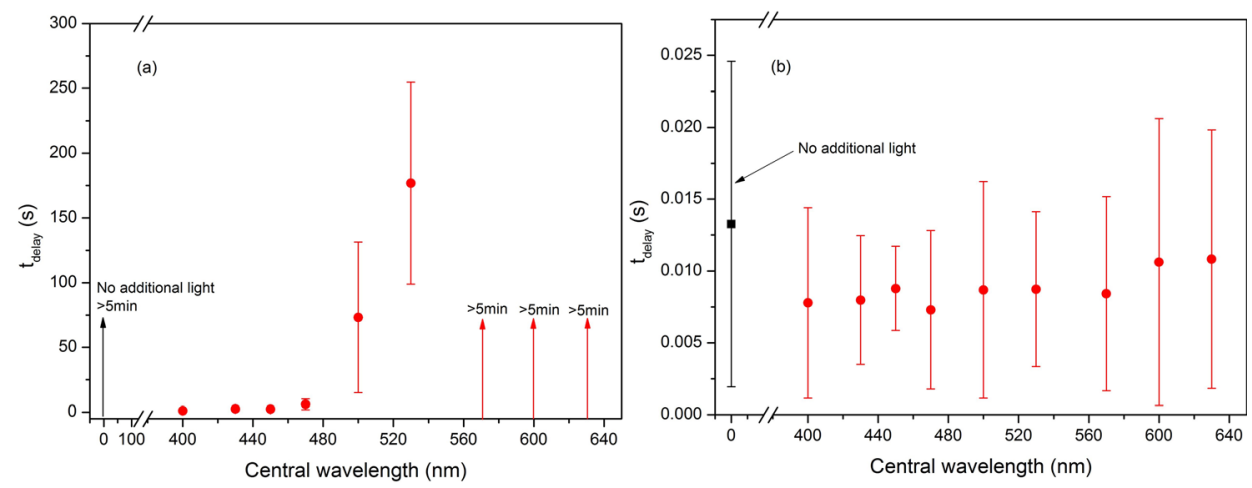

FIG. 7. The ignition delay times for (a) 99.99\% $\mathrm{N}_{2}$ and (b) $99.99 \% \quad \mathrm{O}_{2}$ when light with different central wavelengths is used at a total gas pressure of $10^{4} \mathrm{~Pa}$. Other parameters are the same as in Fig. 6. oxygen so that oxygen molecules attach free electrons generated by the extra light illumination.

As can be seen in Fig. 6, the ignition delay times for the case with $1 \%$ oxygen addition reveal some difference. In order to investigate the effect of the visible light on nitrogen and oxygen, $99.99 \%$ nitrogen is chosen for Fig. 7(a) and 99.99\% oxygen is chosen for Fig. 7(b).

It is hard to breakdown pure nitrogen without light as can be seen from Fig. 7(a). Light irradiation with the central wavelengths from 400to $530 \mathrm{~nm}$ help decrease the breakdown delay time significantly. However, light with the central wavelengths higher than $530 \mathrm{~nm}$ play a less important role in the ignition stage. In Fig. 7(b), it is interesting that the delay time is much shorter than for pure He in Fig. 5(b) and for pure $\mathrm{N}_{2}$ gases in Fig. 7(a). Importantly, light has almost no effect on the delay time for pure oxygen.

Comparing with Figs. 6(b) and 7(b), it has been found that the effect of addition of $\mathrm{O}_{2}$ (without illumination) is unexpected. While the additive of $1 \% \mathrm{O}_{2}$ to helium leads to the increase of $t_{\mathrm{d}}$. But in pure $\mathrm{O}_{2}, t_{\mathrm{d}}$ is several orders less than in $\mathrm{He}$. In order to better understand the effect of $\mathrm{O}_{2}$ on $t_{\mathrm{d}}$, the ignition delay time versus oxygen percentage are measured as shown in Fig. 8.

As can be seen from Fig. 8, the mixture gas is difficult to be ignited with $1 \%-20 \% \mathrm{O}_{2}$ at pressure of $10^{4} \mathrm{~Pa}$ as the ignition delay time is longer than $5 \mathrm{~min}$, but it is much easier

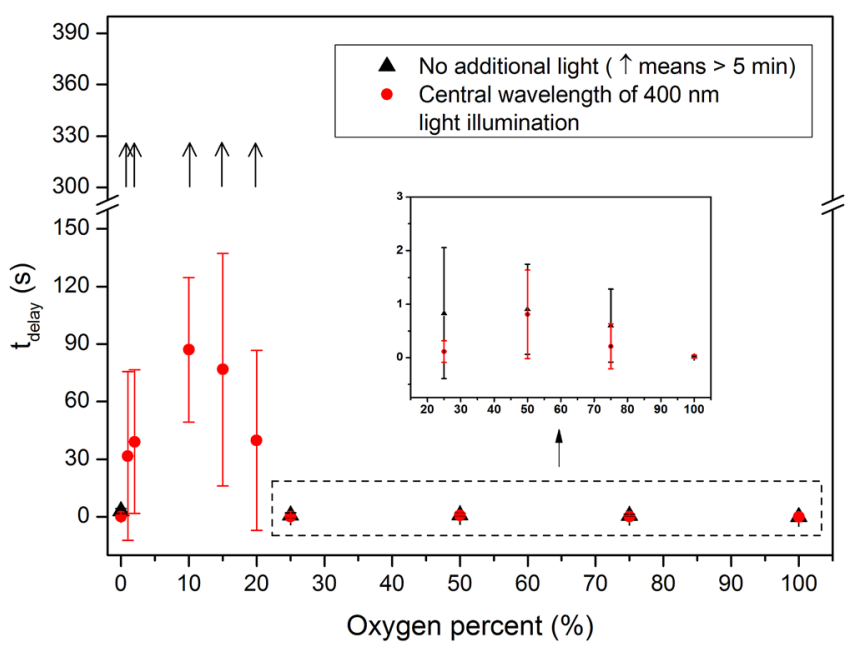

FIG. 8. The ignition delay times versus oxygen percentage with the central wavelength of $400 \mathrm{~nm}$ light illuminated on or off (gas pressure of $10^{4} \mathrm{~Pa}$; voltage parameters are the same as in Fig. 6). to be ignited with either less than $1 \%$ or more than $20 \%$ of $\mathrm{O}_{2}$. Thus, the reaction process in the plasma system with different $\mathrm{O}_{2}$ percent must have changed. With low oxygen concentration $(<1 \%)$, Penning processes $\left(\mathrm{He}^{*}+\mathrm{O}_{2} \rightarrow \mathrm{He}+\right.$ $\left.\mathrm{O}_{2}^{+}+\mathrm{e} ; \mathrm{He}^{*}+\mathrm{O}_{2}(1 \mathrm{D}) \rightarrow \mathrm{He}+\mathrm{O}_{2}^{+}+\mathrm{e} ; \mathrm{He}^{*}+\mathrm{O} \rightarrow \mathrm{He}+\mathrm{O}^{+}+\mathrm{e}\right)$ play a significant role, and the gas can be easily ignited. With 1\%-20\% oxygen, the electron attachment of oxygen molecule plays the dominate role, which makes the gas difficult to breakdown and this is consistent with that of atmospheric pressure. ${ }^{18}$ When the $\mathrm{O}_{2}$ percentage is further increased to more than $20 \%$, the difference of $t_{\mathrm{d}}$ with and without the light illumination become smaller, which might be explained as following: the increasing role of the process $\left(\mathrm{e}+\mathrm{O}_{2} \rightarrow \mathrm{O}_{2}(1 \mathrm{D})+\mathrm{e}\right)$ make the density of $\mathrm{O}_{2}(1 \mathrm{D})$ increase. Then the process $\left(\mathrm{O}^{-}+\mathrm{O}_{2}(1 \mathrm{D}) \rightarrow \mathrm{O}_{3}+\mathrm{e}\right)$ is enhanced which supply enough seed electrons for ignition.

\section{DISCUSSION}

The most common method for producing the electrical breakdown, generating and sustaining of low temperature plasma, is applying high electric field to a neutral gas. Any volume of neutral gas always contains low density of electrons and ions that are, for example, the result of the interaction of cosmic rays or environment radiation with the neutral gas. These free charge carriers are accelerated by the electric field, colliding with atoms/molecules in the gas and with the electrode surfaces, and new charged particles may be created. This leads to an avalanche of charged particles and to the initiation of electrical breakdown in the gas. When the electric field in the inter electrode space $E$ is sufficiently high to create the multiplication of the electrons and ions, the avalanche appears. If this multiplication creates a sufficient number of electrons and ions, it will lead to the electrical breakdown. However, if the process of free charge species losses is dominated, the avalanche multiplication will cease. The necessary conditions for gas breakdown are the sufficient high electrical field and enough number of effective electrons, but how the seed electrons develop into the effective electrons is probabilistic event. ${ }^{19}$ As the breakdown delay time is the main focus of this work, we relate the process of gas breakdown to the applied voltage. The breakdown delay time $t_{\mathrm{d}}$ includes the statistical time delay $\left(t_{\mathrm{s}}\right)$ and the formation time $\left(t_{\mathrm{f}}\right)$, i.e., $t_{\mathrm{d}}=t_{\mathrm{s}}+t_{\mathrm{f}}$. The period of time between the application of voltage and the electron generation is defined as the statistical time delay $t_{\mathrm{s}}$. Moreover, the 

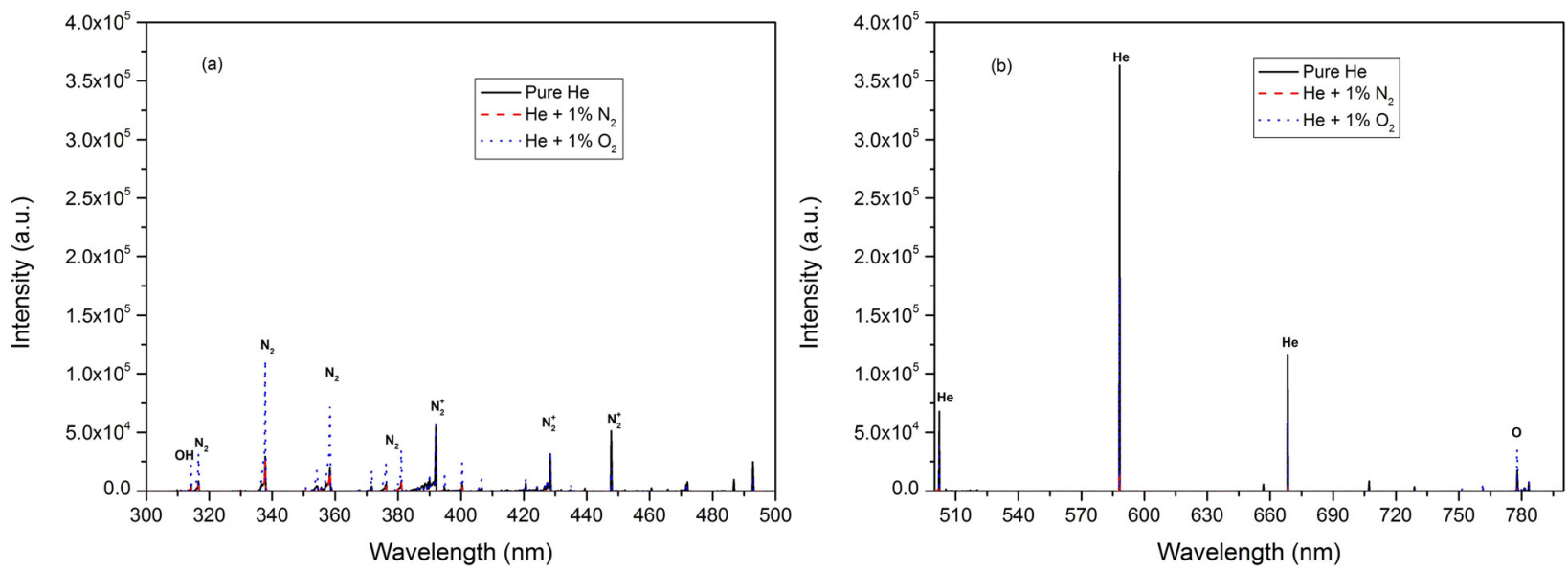

FIG. 9. Optical emission spectra of pure $\mathrm{He}$ and $\mathrm{He}+1 \% \mathrm{~N}_{2}$ and $\mathrm{He}+1 \% \mathrm{O}_{2}$ gas mixtures.

breakdown process can only be initiated when the electrons appear in the intense electric field region. That is the reason why the visible light illumination is most effective when it is focused on the HV electrode. When the electrons appear, the avalanche may form in the intense electric field region. This process creates a sufficient number of electrons and ions and it takes certain time described as the formation time $t_{\mathrm{f}}$. Under conditions of this work, the statistical time delay $t_{\mathrm{s}}$ is much larger than the formation time $t_{\mathrm{f}}{ }^{19}$ Thus, the variation of the $t_{\mathrm{d}}$ is mainly affected by the statistical time delay $t_{\mathrm{s}}$. In this way, the reason for the visible light reducing $t_{\mathrm{d}}$ is to supply free electrons during the ignition stage. The next problem is to identify the possible mechanisms of how visible light supplies free electrons for ignition. There are two distinctive possibilities for the free electrons generation by visible light.

One plausible mechanism is the optically stimulated exoelectron emission (OSEE), which arises upon radiation with the wavelength exceeding the photoelectric threshold. OSEE is a particular case of exoelectron emission (EEE), which is low-temperature emission of electrons from a solid surface subjected to various external effects. ${ }^{20}$ These effects are considered as preliminary excitation of the electron emitter. In Ref. 21, this excitation was performed by mechanical grinding of the electrode, which leads to the formation of microscopic charged cracks in the oxide layer. Under the gas-discharge conditions, the emitter (wall) could be excited as charging of the wall upon its contact with the plasma during the tube heating or during the previous breakdown. It is very challenging to measure the EEE current as it is very low, typically ranging from $10^{-18}$ to $10^{-11} \mathrm{~A}^{20}$

The other possibility is the presence of nitrogen species, which can be vibrationally excited by photons. Though pure helium with $99.999 \%$ purity was used, typically at least $5 \mathrm{ppm}$ nitrogen impurity is present inside the chamber, while the impurity level can be even higher when gas leakage is taken into consideration. The optical emission spectra of the discharges in pure helium, helium with $1 \% \mathrm{~N}_{2}$, and helium with $1 \% \mathrm{O}_{2}$ of Section III B are presented in Fig. 9. From Fig. 9, one can always note $\mathrm{N}_{2}$ and $\mathrm{N}_{2}{ }^{+}$peaks in pure helium, helium with $1 \% \mathrm{~N}_{2}$, and helium with $1 \% \mathrm{O}_{2}$, which confirms the presence of $\mathrm{N}_{2}$ impurity in pure helium. Because the energy for the nitrogen vibration excitation is about $1.7-3.5 \mathrm{eV},{ }^{17}$ the light with the central wavelengths of $400-630 \mathrm{~nm}$ facilitates the vibration excitation as the corresponding energies range from 3.105 to $1.97 \mathrm{eV}$. Nitrogen molecules are vibrationally excited by the narrow visible light, which makes the electron impact ionization easier afterwards.

\section{CONCLUSION}

The breakdown delay time $t_{\mathrm{d}}$ is the sum of the statistical delay time $t_{\mathrm{s}}$ (initiating-electron expectation time) and the formation time $t_{\mathrm{f}}$ (time during which the ionization processes develop). In this work, the variation of $t_{\mathrm{d}}$ is mainly related to the change of $t_{\mathrm{s}}$. Several interesting conclusions can be made as follows.

First, the visible light reduces the breakdown delay time effectively. The effect is inversely related to the wavelength of the additional light. The visible light provides free electrons for the discharge. The production mechanism is still unclear and there are two possible mechanisms. One of the mechanisms is the OSEE from the inner surface of the discharge tube wall and the other is the vibration excitation of the impurity nitrogen molecules. However, in order to obtain a deeper insight in the free electron generation mechanisms, further studies are needed.

Second, the position of the focused light spot is very important, and the best effect is achieved when it is near the HV electrode. This phenomenon is due to the fact that the ionization processes in a strongly non-uniform electric field initially develop near the electrode rather than throughout the entire tube. As a result, the ionization wave front forms at the high-voltage electrode and then propagates through the tube.

Third, the tube material and size are important for enhancing the effect. The jet using quartz tube is much easier to be ignited with the visible light illuminated on. And with the same wall thickness, the larger inner diameter makes the effect stronger.

Finally, the visible light becomes much more effective with increasing the frequency and the pulse width of the voltage. However, due to the electronegativity of oxygen, the ignition delay is significantly affected by the oxygen 
concentration. The visible light can reduce the breakdown delay time effectively when the gas is mixed with $1 \%-20 \%$ $\mathrm{O}_{2}$. Unlike discharge at the atmospheric pressure, the gas with larger than $20 \%$ oxygen can be breakdown. But the effect of visible light on the delay time $t_{\mathrm{d}}$ is not obvious.

\section{ACKNOWLEDGMENTS}

This work was partially supported by the National Natural Science Foundation (Grant Nos. 51277087, 51477066, 51625701, and 11505070), CSIRO Science Leadership Scheme, and the Australian Research Council.

${ }^{1}$ X. Lu, G. V. Naidis, M. Laroussi, and K. Ostrikov, Phys. Rep. 540, 123 (2014).

${ }^{2}$ G. C. Kim, G. J. Kim, S. R. Park, S. M. Jeon, H. J. Seo, F. Iza, and J. K. Lee, J. Phys. D: Appl. Phys. 42, 032005 (2009).

${ }^{3}$ X. Lu, G. V. Naidis, M. Laroussi, S. Reuter, D. B. Graves, and K. Ostrikov, Phys. Rep. 630, 1 (2016).

${ }^{4}$ M. Keidar and I. I. Beilis, J. Appl. Phys. 106, 103304 (2016).

${ }^{5}$ G. Park, H. Lee, G. Kim, and J. K. Lee, Plasma Process. Polym. 5, 569 (2008).

${ }^{6}$ H. S. Uhm, E. H. Choi, and G. S. Cho, Appl. Phys. Lett. 78, 592 (2001).
${ }^{7}$ Q. Lu, D. Liu, Y. Song, R. Zhou, and J. Niu, Plasma Process. Polym. 11, 1028-1036 (2014).

${ }^{8}$ X. L. Deng, A. Y. Nikiforov, P. Vanraes, and C. Leys, J. Appl. Phys. 113, 023305 (2013).

${ }^{9}$ Y. Song, D. Liu, L. Ji, W. Wang, P. Zhao, C. Quan, J. Niu, and X. Zhang, Plasma Process. Polym. 9, 17-21 (2012).

${ }^{10}$ S. Wu, X. Lu, and Y. Pan, Curr. Appl. Phys. 13, S1 (2013).

${ }^{11}$ D. Breden, K. Miki, L. L. Raja, D. Breden, K. Miki, and L. L. Raja, Appl. Phys. Lett. 99, 111501 (2011).

${ }^{12}$ G. V. Naidis, J. Phys. D: Appl. Phys. 44, 215203 (2011).

${ }^{13}$ S. Yu, X. Pei, Q. Hasnain, L. Nie, and X. Lu, Phys. Plasmas 23, 023510 (2016).

${ }^{14}$ L. Nie, L. Chang, Y. Xian, and X. Lu, Phys. Plasmas 23, 93518 (2016).

${ }^{15}$ M. M. Pejović, G. S. Ristić, and Z. Lj. Petrović, J. Phys. D: Appl. Phys. 32, 1489 (1999).

${ }^{16}$ A. I. Shishpanov, Y. Z. Ionikh, and A. V. Meshchanov, Opt. Spectrosc. 120, 871 (2016).

${ }^{17}$ A. Fridman, Plasma Physics and Engineering (CRC Press, 2011).

${ }^{18}$ D. X. Liu, M. Z. Rong, X. H. Wang, F. Iza, M. G. Kong, and P. Bruggeman, Plasma Process. Polym. 7, 846 (2010).

${ }^{19}$ M. M. Pejović, G. S. Ristić, and J. P. Karamarković, J. Phys. D: Appl. Phys. 35, R91 (2002).

${ }^{20}$ R. I. Mints, I. I. Mil'man, and V. I. Kryuk, Sov. Phys. Usp. 19, 697 (1976).

${ }^{21}$ Y. Gosho and A. Harada, J. Phys. D: Appl. Phys. 16, 1047 (1983). 